\title{
ARIA 2016 executive summary: Integrated care pathways for predictive medicine throughout the life cycle in Argentina
}

\section{Resumen ejecutivo de ARIA 2016: vías integradas de atención para la medicina predictiva a lo largo del ciclo de vida en Argentina}

Juan Carlos Ivancevich, ${ }^{1}$ Hugo Neffen, ${ }^{2}$ Mario E. Zernotti, ${ }^{3}$ Estrella Asayag, ${ }^{4}$ Ariel Blua, ${ }^{5}$ Alberto Cicerán, ${ }^{6}$ Edgardo J. Jares, ${ }^{7}$ Alberto J. Lavrut, ${ }^{8}$ Jorge F. Máspero, ${ }^{9}$ Ioana Agache, ${ }^{10}$ Claus Bachert, ${ }^{11}$ Anna Bedbrook, ${ }^{12}$ Giorgio W. Canonica, ${ }^{13}$ Thomas B. Casale,${ }^{14}$ Álvaro A. Cruz,,${ }^{15}$ Wytske J. Fokkens, ${ }^{16}$ Peter W. Hellings, ${ }^{17}$ Boleslaw Samolinski, ${ }^{18}$ Jean Bousquet ${ }^{19}$

\begin{abstract}
The ARIA initiative was started during a World Health Organization workshop in 1999. The initial goals were to propose a new classification for allergic rhinitis, to promote the concept of multimorbidity in asthma and rhinitis and to develop guidelines with stakeholders for world-wide use. ARIA is now focused on the implementation of emerging technologies for individualized and predictive medicine. MASK: MACVIA-Aria Sentinel Network uses mobile technology to develop care pathways that enable management by a multidisciplinary group or by patients themselves. An App for iOS and Android uses a visual analogue scale to assess symptom control and work productivity, as well as a clinical decision support system; it is associated with an interoperable tablet for health professionals. The escalation strategy uses recommendations of the European Innovation Partnership on Active and Healthy Ageing. The aim of ARIA's new approach is to provide an active and healthy life to people affected by rhinitis, regardless of age, gender or socioeconomic status, in order to reduce social and health inequalities caused by the disease.
\end{abstract}

Keywords: ARIA initiative; Rhinitis; ICT; EIP on AHA; Mobile technology; Airways ICPs

Este artículo debe citarse como: Ivancevich JC, Neffen H, Zernotti ME, Asayag E, Blua A, Cicerán A, Jares EJ, Lavrut AJ, Máspero JF, Agache I, Bachert C, Bedbrook A, Canonica GW, Casale TB, Cruz ÁA, Fokkens WJ, Hellings PW, Samolinski B, Bousquet J. Resumen ejecutivo de ARIA 2016: vías integradas de atención para la medicina predictiva a lo largo del ciclo de vida en Argentina. Rev Alerg Mex. 2017;64(3):298-308

${ }^{1}$ Facultad de Medicina, Universidad del Salvador. Buenos Aires, Argentina

${ }^{2}$ Centro de Alergia, Inmunología y Enfermedades Respiratorias. Santa Fe, Argentina

${ }^{3}$ Sanatorio Allende, Departamento de Otorrinolaringología. Córdoba, Argentina

${ }^{4}$ Sociedad Argentina de Alergia e Inmunopatología. Buenos Aires, Argentina

${ }^{5}$ Asociación Argentina de Medicina Respiratoria. Buenos Aires, Argentina

${ }^{6}$ Federación Argentina de Sociedades de Otorrinolaringología. Buenos Aires, Argentina
${ }^{7}$ Sociedad Latinoamericana de Alergia, Asma e Inmunología. Buenos Aires, Argentina

${ }^{8} \mathrm{Hospital}$ General de Niños Pedro de Elizalde-Casa Cuna, Unidad de Alergia. Buenos Aires, Argentina

${ }^{9}$ Asociación Argentina de Alergia e Inmunología Clínica. Buenos Aires, Argentina

${ }^{10}$ Universidad de Transilvania, Facultad de Medicina, Departamento de Alergia e Inmunología Clínica. Brasov, Rumanía

${ }^{11}$ Hospital Universitario de Ghent, Departamento de Otorrinolaringología, Laboratorio de Investigación de Vías Aéreas Superiores. Ghent, Bélgica 


\section{Resumen}

La iniciativa ARIA (Rinitis Alérgica y su Impacto en el Asma) se inició durante un taller de la Organización Mundial de la Salud en 1999. Los objetivos iniciales fueron proponer una nueva clasificación de rinitis alérgica, promover el concepto de multimorbilidad en asma y rinitis y desarrollar guías con todas las partes interesadas para su en todos los países y poblaciones. ARIA, difundida e implementada en más de 70 naciones, ahora se centra en la implementación de tecnologías emergentes para la medicina individualizada y predictiva. MASK (MACVIA [Contre les Maladies Chroniques pour un Vieillissement Actif] Aria Sentinel Network) utiliza la tecnología móvil para desarrollar vías de atención que permitan el manejo de la rinitis y del asma por un grupo multidisciplinario o por los propios pacientes. Una aplicación (app) para iOS y Android está disponible en 20 países y 15 idiomas; utiliza una escala analógica visual para evaluar el control de los síntomas y la productividad del trabajo, así como un sistema de apoyo para las decisiones clínicas. Se asocia con una tabla interoperable (que permite intercambiar información) para médicos y otros profesionales de la salud. La estrategia de escalamiento utiliza las recomendaciones de la Asociación Europea de Innovación para el Envejecimiento Activo y Saludable. El objetivo del nuevo enfoque ARIA es proporcionar una vida activa y saludable a las personas afectadas por la rinitis, cualquiera que sea su edad, sexo o condición socioeconómica, con el fin de reducir las desigualdades sociales y de salud causadas por la enfermedad.

Palabras clave: Iniciativa ARIA; Rinitis; Tecnologías de la Información y la Comunicación; Asociación Europea de Innovación para un Envejecimiento Activo y Saludable; Tecnología móvil; Vías de atención integradas para las enfermedades de las vías respiratorias

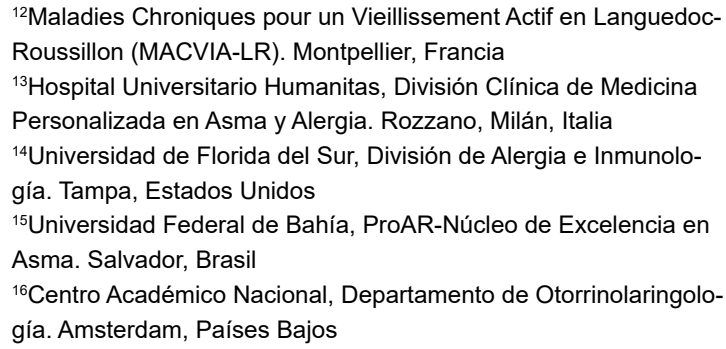

\section{Abreviaturas y siglas}

Airways ICPs, vías de atención integradas para las enfermedades de las vías respiratorias

ARIA, Rinitis Alérgica y su Impacto en el Asma

CDSS, Sistema de Apoyo a la Decisión Clínica

DG, Dirección General

ECA, Ensayo controlado aleatorizado

EIP sobre AHA, Asociación Europea de Innovación para un Envejecimiento Activo y Saludable

EVA, Escala analógica visual

FP, Programa Marco

GARD, Alianza Global de la OMS contra las Enfermedades Respiratorias Crónicas

\begin{abstract}
${ }^{17}$ Hospital Universitario de Leuven, División Clínica de Otorrinolaringología, Cirugía de Cabeza y Cuello. Leuven, Bélgica

${ }^{18}$ Universidad Médica de Varsovia, Departamento de Prevención de Peligros Ambientales y Alergología. Varsovia, Polonia ${ }^{19}$ Maladies Chroniques pour un Vieillissement Actif en LanguedocRoussillon (MACVIA-LR). Montpellier, Francia
\end{abstract}

Correspondencia: Juan Carlos Ivancevich. ivancev@gmail.com

Recibido: 2017-06-18

Aceptado: 2017-06-30 


\section{Introducción}

Los pacientes, los clínicos y otros profesionales de la salud se enfrentan con diversas opciones de tratamiento para el manejo de la rinitis alérgica, lo que contribuye a una considerable variabilidad en la práctica clínica y los pacientes a menudo expresan su insatisfaccion con su tratamiento. La iniciativa ARIA (Rinitis Alérgica y su Impacto en el Asma) comenzó durante un taller de la Organización Mundial de la Salud en 1999 y fue publicada en 2001. Su objetivo fue proporcionar una guía para el diagnóstico y tratamiento de la multimorbilidad de la rinitis alérgica y el asma. ${ }^{1,2}$ En 2008, ARIA se actualizó utilizando el mismo sistema de recomendaciones. ${ }^{1,3}$ En su revisión de 2010, ARIA se constituyó en la primera guía de enfermedades respiratorias crónicas en adoptar el enfoque GRADE (Grading of Recommendation, Assessment, Development and Evaluation), metodología avanzada de evaluación de evidencias. ${ }^{4,5}$

ARIA, difundida e implementada en más de 70 países, ${ }^{6}$ actualmente se centra en la implementación de tecnologías emergentes para la medicina individualizada y predictiva. MASK (MACVIA [Contre les MAladies Chroniques pour un VIeillissement Actif]-ARIA Sentinel Network) utiliza la tecnología móvil para desarrollar vías de atención que permitan el manejo de la rinitis y el asma por un grupo multidisciplinario o por los propios pacientes. ${ }^{7,8}$

El objetivo del nuevo enfoque ARIA es proporcionar una vida activa y saludable a las personas afectadas por rinitis durante todo su ciclo vital, independientemente de su sexo o condición socioeconómica, con el fin de reducir las inequidades de la salud y sociales causadas por la enfermedad.

Vías de atención integradas para las enfermedades respiratorias: Airways ICPs. La agenda política de ARIA 2016 En 2012, la Comisión Europea lanzó la Asociación Europea para la Innovación en Envejecimiento Activo y Saludable (EIP en AHA, DG Santé y DG CONNECT). El objetivo era mejorar la competitividad de la Unión Europea y afrontar los desafíos sociales del envejecimiento mediante la investigación y la innovación. ${ }^{9}$ El Plan de Acción B3 está dedicado a la ampliación y replicación de modelos exitosos e innovadores de atención integral para las enfermedades crónicas en pacientes de edad avanzada; para ello se utilizaron enfermedades respiratorias crónicas como proyecto piloto.

Airways ICPs (Vías integradas de atención para las enfermedades respiratorias) es la herramienta de implementación del proyecto; su principal actividad es el desarrollo de vías de atención multisectoriales para la rinitis, el asma y sus múltiples morbilidades, mediante tecnologías emergentes en medicina predictiva a lo largo del ciclo de vida del paciente..$^{7,810} \mathrm{El}$ objetivo es reducir la carga crónica de las enfermedades respiratorias, la mortalidad y la multimorbilidad, manteniendo al mismo tiempo la calidad de vida. ${ }^{711}$ El plan de acción de Airways ICPs se ha implementado y ampliado a escala mundial. ${ }^{12}$ Además, Airways ICPs es un proyecto de demostración de la Alianza Global contra las Enfermedades Respiratorias Crónicas de la Organización Mundial de la Salud (GARD) (Figura 1). ARIA 2016 es un componente importante de Airways ICPs. ${ }^{13}$

De las guías a las vías de atención integradas: MACVIA-ARIA Sentinel NetworK (MASK) Aun cuando la rinitis alérgica afecta negativamente la vida social, escolar y productividad laboral, ${ }^{3}$ un gran número de pacientes con rinitis alérgica autocontrolan su enfermedad y se autorrecetan debido al acceso a numerosos medicamentos de venta libre; $;^{14,15.16,17}$ la mayoría de los pacientes que consultan a los médicos generalistas o especialistas padecen una rinitis moderada a grave. ${ }^{18,19,20}$ Además, los pacientes también pueden optar por la medicina alternativa o la inmunoterapia alérgeno específica. ${ }^{21}$ Es por ello que las vías de atención integradas (IPCs) deberían considerar un enfoque multidisciplinario que incluya el automanejo, como fuera propuesto por Airways ICPs (Figura 2).

Las IPCs son planes multidisciplinarios estructurados que detallan pasos clave en el cuidado del paciente. Promueven la adaptación de las guías a protocolos locales y su consecuente aplicación en la práctica clínica. Las IPCs se diferencian de las guías de práctica clínica en que son utilizadas por un equipo multidisciplinario y se enfocan en la calidad y coordinación de la atención; necesitan tener un mecanismo para registrar las variaciones o desviaciones en el plan de tratamiento.

En las recomendaciones de ARIA, las variaciones o desviaciones en el plan de tratamiento deben ser evaluadas. La gravedad de la enfermedad está asociada con varios efectos sobre la sa- 


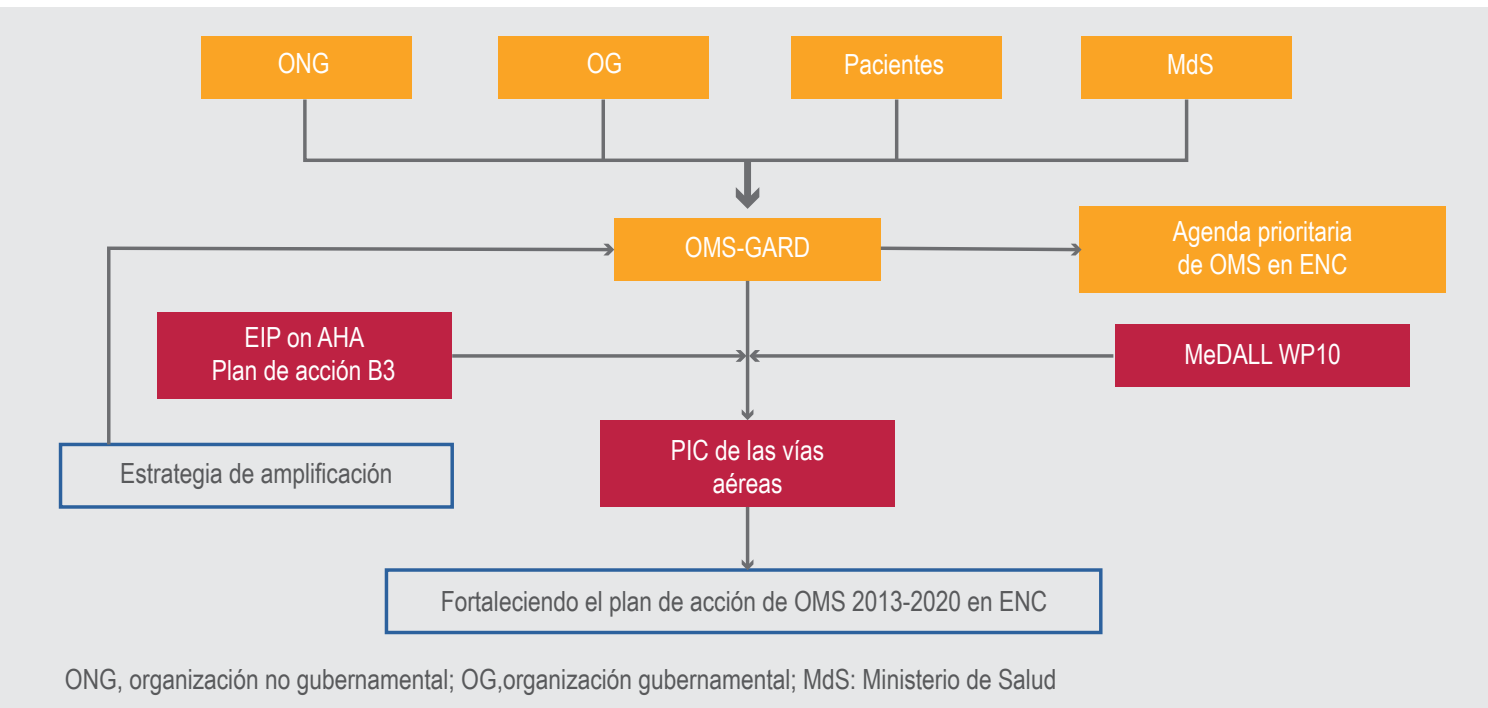

Figura 1. Pasos de tratamiento integrado de las vías aéreas: interacciones entre la Unión Europea y la Organización Mundial de la Salud. ${ }^{11}$

lud, incluyendo la calidad de vida. ${ }^{18,19,20}$ La clasificación de rinitis (intermitente/grave-persistente) es un indicador importante de la multimorbilidad del asma (solo en algunos estudios), ${ }^{22}$ así como de la duración y eficacia del tratamiento de la rinitis alérgica. ${ }^{23}$ Sin embargo, la mayoría de los pacientes recibe combinaciones de antihistamínicos orales y corticoides nasales, las cuales no están suficientemente evaluadas en guías que usen una metodología apropiada. ${ }^{24}$

\section{Enfoque simple para evaluar el control de la rinitis alérgica}

ARIA condujo el cambio de gravedad de los síntomas al control de la enfermedad en rinitis alérgica como guía de las decisiones terapéuticas e incluyó una escala visual analógica (EVA) como un lenguaje común validado del control de la rinitis alérgica, categorizando el control mediante puntos de corte en la EVA. Incorporó la EVA en una aplicación interactiva para pacientes (Diario de alergia ARIA) y profesionales de la salud (Diario de alergia complementario ARIA $)^{8,25,26}$ que integra todos los datos en las IPCs, ${ }^{25}$ y desarrolló un sistema de apoyo para decisiones clínicas.

Los síntomas de la rinitis alérgica tienen una variación diaria y se necesita un régimen terapéuti- co personalizado a través del tiempo. Los pacientes, cuidadores o profesionales de la salud deben utilizar un sistema de puntuación de síntomas simple rápidamente sensible a los cambios. MACVIA-ARIA ha desarrollado un algoritmo basado en una EVA simple llamada ARIA CDSS (Sistema de Soporte de Decisión Clínica ARIA), que utiliza una puntuación para guiar las decisiones respecto al tratamiento. ${ }^{27}$

\section{Herramientas de MASK: Diario de alergia y Diario de alergia complementario}

El Diario de alergia ARIA se encuentra disponible en forma gratuita para pacientes de Australia, Brasil, Canadá, México, Suiza y 15 países de la Unión Europea, en 15 idiomas (adaptado culturalmente y compatible legalmente). Las aplicaciones complementarias para los profesionales de la salud también están disponibles sin costo. ${ }^{26}$

\section{Cuestionarios}

Al registrarse, los usuarios del Diario de alergia ARIA completan cuestionarios simples sobre asma, rinitis y el impacto de la enfermedad (globalmente, en el trabajo, en la escuela, en las actividades diarias y en el sueño) (Cuadro 1). El estudio piloto, que incluye alrededor de 5000 usuarios (9\% mayores de 60 años), indica que estas preguntas son fáciles 


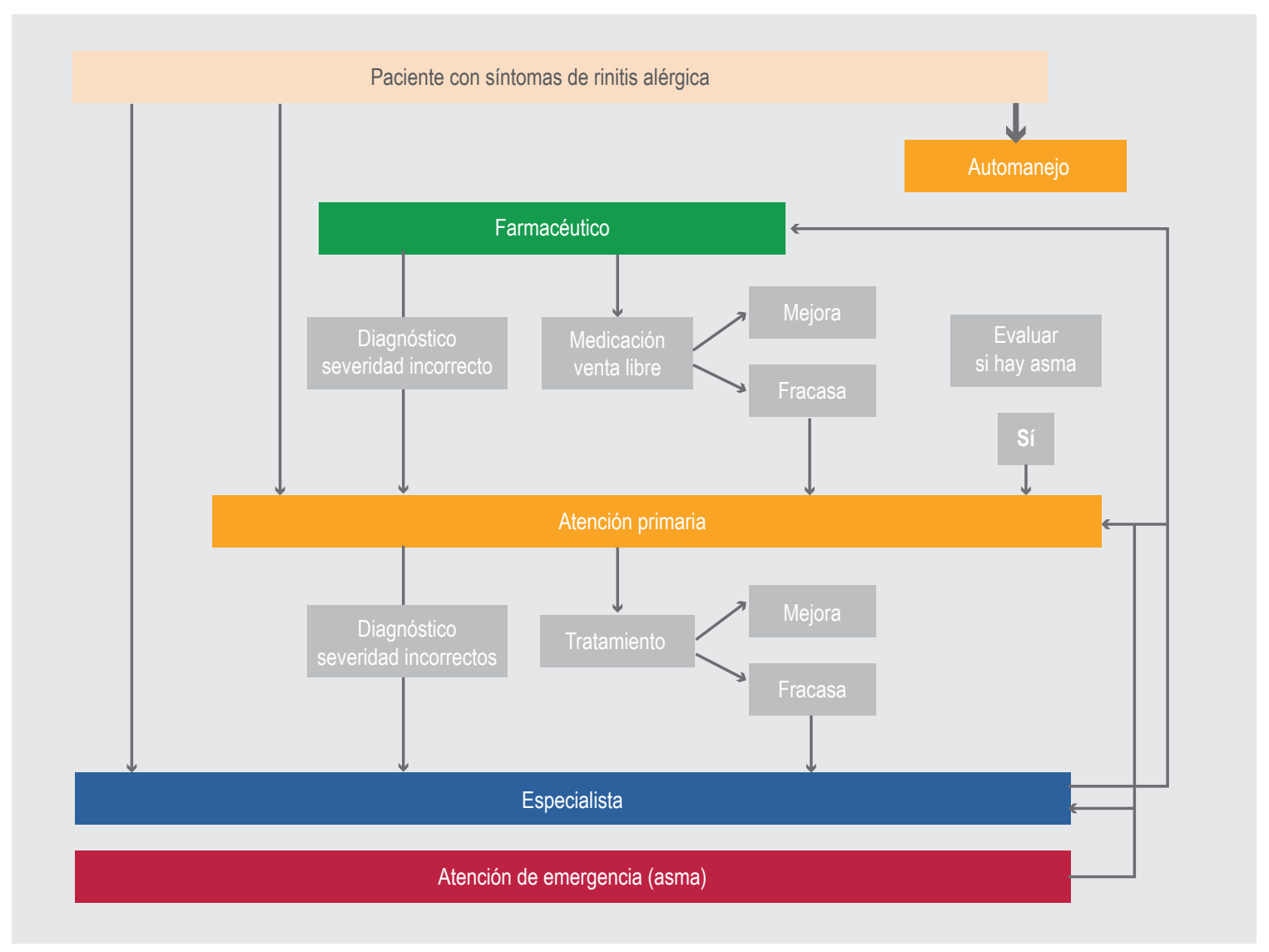

Figura 2. Sendero de cuidado multisectorial para la rinitis alérgica. ${ }^{25}$

de responder y pueden ayudar a estratificar a los pacientes con rinitis. ${ }^{28}$

Además, se aplican 2 cuestionarios específicos cada semana para evaluar el impacto de la enfermedad en la calidad de vida (EQ-5D $)^{29}$ y la productividad en el trabajo (WPAI-AS). ${ }^{30}$

\section{Tratamientos recibidos}

Una lista de todos los tratamientos disponibles para el asma, la conjuntivitis y la rinitis está incluida en el Diario de alergia ARIA y los usuarios seleccionan el o los tratamientos que están tomando. Pueden seleccionar múltiples tratamientos y actualizar la información cuando hay cambios al respecto (Figura 3).

La lista se ha personalizado para los 20 países en los que está disponible el Diario de alergia ARIA. También se solicita información sobre la inmunoterapia específica con alérgenos el día del primer uso.

\section{EVA diarias}

Los usuarios geolocalizados evalúan su control diario de los síntomas a través de la funcionalidad de la pantalla táctil en su teléfono inteligente después de contestar 5 EVA consecutivas (síntomas globales debido a enfermedades alérgicas, rinitis, conjuntivitis, asma y productividad laboral) (Figura 4).

\section{Sistema de apoyo para las decisiones clínicas}

La MASK CDSS se incorpora a una aplicación para los profesionales de la salud (Diario complementario de alergia ARIA). Se trata de un algoritmo basado en una EVA para orientar a los médicos en la selección de los medicamentos para los pacientes con rinitis alérgica y a estratificar la gravedad de la enfermedad. ${ }^{27}$ Utiliza un enfoque simple de aumento/disminución individualizado de la farmacoterapia. 
Nuevos conceptos en multimorbilidad. Estratificación de pacientes con alergia o asma grave

A pesar de los grandes avances en la comprensión de la rinitis alérgica, los tratamientos no son eficaces en todos los pacientes. ${ }^{31}$ La intención es proporcionar intervenciones terapéuticas más efectivas adaptadas al individuo mediante la estratificación del paciente con MASK.
Los estudios de cohorte a largo plazo desde el nacimiento son esenciales para comprender el curso de la historia natural de las enfermedades alérgicas (incluidos el asma y la rinitis) a lo largo de la vida y la compleja interacción entre los genes y el medio ambiente. ${ }^{32}$ BAMSE (Barn Allergi Miljö Stockholm Epidemiologi Projektet) $)^{33}$ y MeDALL (Mechanisms of the Development of Allergy; $)^{34}$ identificaron un fenotipo de alergia raro pero

Cuadro 1. Cuestionario inicial

$\mathrm{P} 1$ : Yo tengo rinitis: Sí/No

P2: Yo tengo asma: Sí/No

P3: Mis síntomas (marcar)

- Rinorrea

- Prurito nasal

- Estornudos

- Congestión (nariz tapada)

- Ojos rojos

- Picazón ocular

- Lagrimeo (ojos llorosos)

P4: Cómo me afectan mis síntomas (marcar)

- Afectan mi sueño

- Restringen (limitan) mis actividades diarias

- Restringen (limitan) mi participación en la escuela o en el trabajo

- Son problemáticos

P5: Medicaciones

P6: ¿Recibe actualmente inmunoterapia (una pequeña dosis de la sustancia a la que es alérgico, usualmente administrada como inyección o colocada bajo su lengua)? Sí/No

Si la respuesta es Sí, contestar P7 y P8

P7: ¿Cual alergia (alérgeno)?

- Pólenes de pastos

- Pólenes de malezas

- Pólenes de árboles

- Otro polen

- Ácaros del polvo

- Animales

- No lo sé

- Agregue cualquier otra alergia

P8: ¿Cómo recibe su tratamiento?

- Inyecciones

- Tabletas sublinguales

- Gotas sublinguales

- Aerosol sublingual

- Otras 

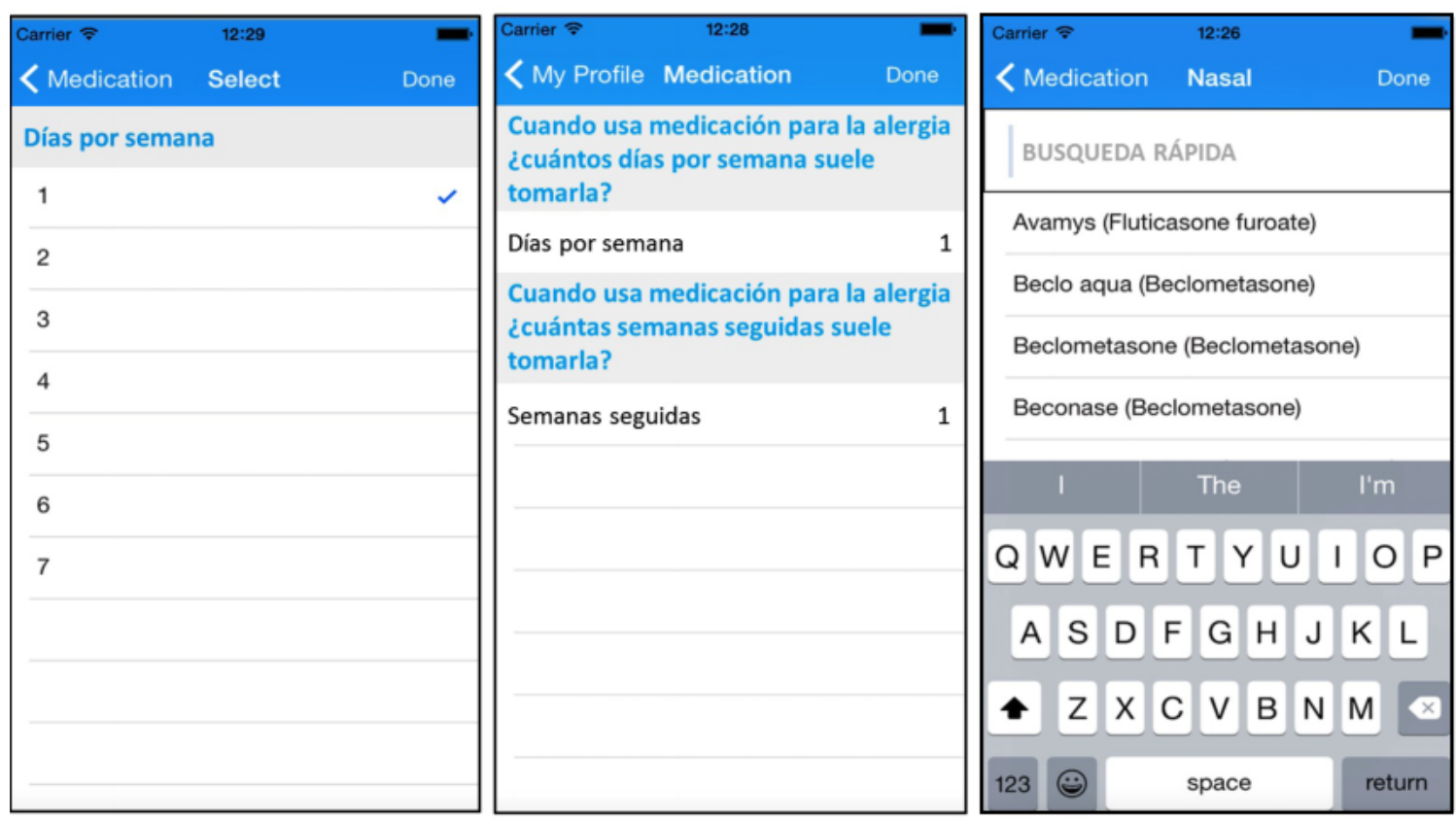

Figura 3. Pantallas sobre medicación.

grave: fenotipo polisensibilizado-multimórbido, confirmado en cohortes de niños y adultos. ${ }^{35,36,37,38}$ Estas investigaciones confirman que los sujetos polisensibilizados y multimórbidos tienen una frecuencia muy alta de síntomas alérgicos, síntomas persistentes a lo largo del tiempo, síntomas más graves de asma o rinitis y niveles más altos de $\operatorname{IgE}$ total y específica.

Tomados en conjunto, estos resultados indican que los pacientes asmáticos no pueden ser manejados apropiadamente sin considerar la multimorbilidad de la rinitis y que los problemas nasales (rinitis o rinosinusitis) son importantes en numerosos pacientes asmáticos no controlados. ${ }^{39,40}$

\section{La multimorbilidad alérgica en los adultos mayores}

La esperada ola epidémica de asma y rinitis en los adultos mayores es un problema insuficientemente reconocido. Los adultos mayores con asma y rinitis tienen síntomas y necesidades de tratamiento específicos. Estos pacientes también sufren de multimorbilidad y se reportan altas tasas de polifarmacia. Las vías de atención integradas para la rinitis y el asma deben cubrir todo el ciclo de vida.
La estrategia de amplificación en Argentina Existe una necesidad urgente de implementar estrategias de amplificación con la finalidad de evitar la fragmentación, mejorar la prestación de asistencia sanitaria, acelerar la implementación de buenas prácticas con base en historias exitosas de costo/efectividad y cumplir con los objetivos de la EIP sobre AHA. ${ }^{9}$ Esta estrategia ya se ha aplicado al plan de acción de las enfermedades respiratorias crónicas. ${ }^{12}$ En Argentina se está instrumentando una estrategia de escalamiento en 3 niveles, con un nivel general que los articula:

- Primer nivel: incorporación de la temática de ARIA y el manejo de guías de diagnóstico y tratamiento específicas en los programas de las carreras de especialización. Esta acción debería implementarse al menos en neumología, alergología y otorrinolaringología, mediante un acuerdo con las autoridades de la Comisión Nacional de Evaluación y Acreditación Universitaria, para que en las carreras se incluyan estos temas.

- Segundo nivel: firmar convenios con las sociedades nacionales de las especialidades generales, 


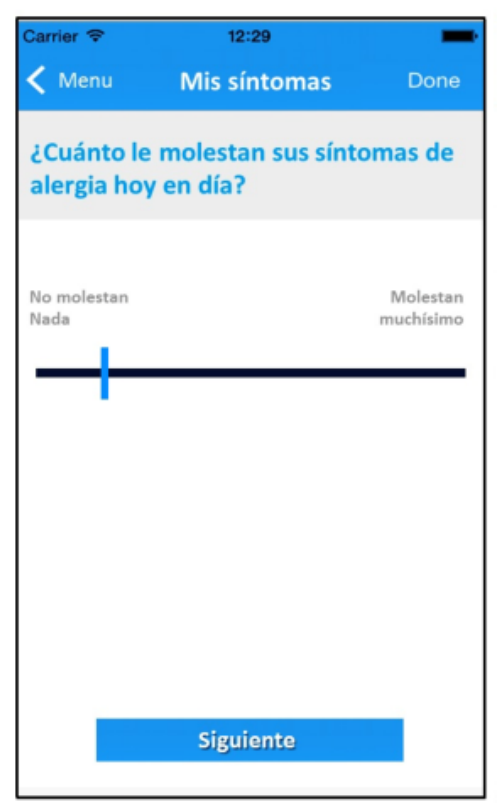

como la Sociedad Argentina de Medicina Interna y la Sociedad Argentina de Pediatría, para que en sus respectivos congresos, simposios o jornadas se incorporen temas de alergia y medicina respiratoria y se divulguen las guías y su implementación.

- Tercer nivel: acuerdos con las sociedades nacionales de especialistas: Asociación Argentina de Alergia e Inmunología Clínica, Sociedad Argentina de Alergia e Inmunopatología, Asociación Argentina de Medicina Respiratoria y Federación Argentina de Sociedades de Otorrinolaringología, para tener espacios de divulgación de la guía y capacitación específica sobre técnicas diagnósticas en alergia, exámenes de función respiratoria, evaluación endoscópica de la vía aérea superior, etcétera.

- Nivel general: en los 3 niveles anteriores incorporar las apps Diario de la alergia ARIA y Diario de alergia complementario ARIA, a fin de adies- trar a los colegas, para que a su vez preparen a los pacientes en el manejo de la aplicación.

\section{Conclusión}

ARIA ha evolucionado desde una guía rigurosamente desarrollada a una estrategia de implementación basada en la tecnología móvil para proporcionar una vida activa y saludable a las personas afectadas por la rinitis, cualquiera que sea su edad, sexo o condición socioeconómica, con el objetivo de reducir las desigualdades sociales y de salud que se producen en el diagnóstico y tratamiento de esta enfermedad tan común.

\section{Financiamiento}

ARIA 2016 recibió financiamiento del Portal de Referencia de Innovación Europea sobre el Envejecimiento Activo y Saludable, MACVIA-Francia, Fondo de Desarrollo y Estructuras de la Unión Europea Languedoc-Roussillon.

\section{Referencias}

1. Shekelle PG, Woolf SH, Eccles M, Grimshaw J. Clinical guidelines: Developing guidelines. BMJ. 1999;318(7183):593-596. DOI: http://dx.doi.org/10.1136/bmj.318.7183.593

2. Bousquet J, Van-Cauwenberge $P$, Khaltaev N. Allergic rhinitis and its impact on asthma. J Allergy Clin Immunol. 2001;108(Suppl 5):147-334. DOI: http://dx.doi.org/10.1067/mai.2001.118891 
3. Bousquet J, Khaltaev N, Cruz AA, Denburg J, Fokkens WJ, Togias A, et al. Allergic Rhinitis and its Impact on Asthma (ARIA) 2008 update (in collaboration with the World Health Organization, GA(2)LEN and AllerGen). Allergy. 2008;63(Suppl 86):8-160. DOI: http://dx.doi.org/10.1111/j.1398-9995.2007.01620.x

4. Brozek JL, Bousquet J, Baena-Cagnani CE, Bonini S, Canonica GW, Casale TB, et al. Allergic Rhinitis and its Impact on Asthma (ARIA) guidelines: 2010 revision. J Allergy Clin Immunol. 2010;126(3):466476. DOI: http://dx.doi.org/10.1016/j.jaci.2010.06.047

5. Padjas A, Kehar R, Aleem S, Mejza F, Bousquet J, Schünemann HJ, et al. Methodological rigor and reporting of clinical practice guidelines in patients with allergic rhinitis: QuGAR study. J Allergy Clin Immunol. 2014;133(3):777-783. DOI: http://dx.doi.org/10.1016/j.jaci.2013.08.029

6. Bousquet J, Schunemann HJ, Samolinski B, Demoly P, Baena-Cagnani CE, Bachert C, et al. Allergic Rhinitis and its Impact on Asthma (ARIA): Achievements in 10 years and future needs. J Allergy Clin Immunol. 2012;130(5):1049-1062. DOI: http://dx.doi.org/10.1016/j.jaci.2012.07.053

7. Bousquet J, Addis A, Adcock I, Agache I, Agusti A, Alonso A, et al. Integrated care pathways for airway diseases (AIRWAYS-ICPS). Eur Respir J. 2014;44(2):304-323. DOI: http://dx.doi. org/10.1183/09031936.00014614

8. Bousquet J, Hellings PW, Agache I, Bedbrook A, Bachert C, Bergmann KC, et al. ARIA 2016: Care pathways implementing emerging technologies for predictive medicine in rhinitis and asthma across the life cycle. Clin Transl Allergy. 2016;6(1):47. DOI: http://dx.doi.org/10.1186/s13601-016-0137-4

9. Bousquet J, Michel J, Standberg T, Crooks G, lakovidis I, Iglesia M. The European Innovation Partnership on Active and Healthy Ageing: the European geriatric medicine introduces the EIP on AHA column. Eur Geriatr Med. 2014;5(6):361-362. DOI: http://dx.doi.org/10.1016/j.eurger.2014.09.010

10. Bousquet J, Anto JM, Berkouk K, Gergen P, Antunes JP, Augé P, et al. Developmental determinants in non-communicable chronic diseases and ageing. Thorax. 2015;70(6):595-597. DOI: http://dx.doi. org/10.1136/thoraxjnl-2014-206304

11. Bousquet J, Barbara C, Bateman E, Bel E, Bewick M, Chavannes NH, et al. AIRWAYS-ICPs (European Innovation Partnership on Active and Healthy Ageing) from concept to implementation. Eur Respir J. 2016;47(4):1028-1033. DOI: http://dx.doi.org/10.1183/13993003.01856-2015

12. Bousquet J, Farrell J, Crooks G, Hellings P, Bel EH, Bewick M, et al. Scaling up strategies of the chronic respiratory disease programme of the European Innovation Partnership on Active and Healthy Ageing (Action Plan B3: Area 5). Clin Transl Allergy. 2016;6(1):29. DOI: http://dx.doi.org/10.1186/s13601-016-0116-9

13. Bousquet J, Bewick M, Cano A, Eklund P, Fico G, Goswami N, et al. Building bridges for innovation in ageing: Synergies between Action Groups of the EIP on AHA. J Nutr Health Aging. 2017;21(1):92-104. DOI: http://dx.doi.org/10.1007/s12603-016-0803-1

14. Kuehl BL, Abdulnour S, O'Dell M, Kyle TK. Understanding the role of the healthcare professional in patient self-management of allergic rhinitis. SAGE Open Med. 2015;3:2050312115595822. DOI: http:// dx.doi.org/10.1177/2050312115595822

15. Bond $\mathrm{C}$, Bonini $\mathrm{S}$, Bousquet $\mathrm{H}$, Bousquet J, Canonica GW, Howarth $\mathrm{P}$, et al. ARIA in the pharmacy: management of allergic rhinitis symptoms in the pharmacy. Allergic rhinitis and its impact on asthma. Allergy. 2004;59(4):373-387. Disponible en: http://www.progetto-aria.it/materiale/aria_in_the_pharmacy. pdf

16. Canonica GW, Triggiani M, Senna G. 360 degree perspective on allergic rhinitis management in Italy: A survey of GPs, pharmacists and patients. Clin Mol Allergy. 2015;13:25. Disponible en: https://www.ncbi. nlm.nih.gov/pmc/articles/PMC4629286/

17. Meltzer EO, Farrar JR, Sennett C. Findings from an online survey assessing the burden and management of seasonal allergic rhinoconjunctivitis in US patients. J Allergy Clin Immunol Pract. 2017;5(3):779-789. DOI: http://dx.doi.org/10.1016/j.jaip.2016.10.010

18. Bousquet J, Annesi-Maesano I, Carat F, Léger D, Rugina M, Pribil C, et al. Characteristics of intermittent and persistent allergic rhinitis: DREAMS study group. Clin Exp Allergy. 2005;35(6):728-732. DOI: http:// dx.doi.org/10.1111/j.1365-2222.2005.02274.x 
19. Bousquet PJ, Devillier P, Tadmouri A, Mesbah K, Demoly P, Bousquet J. Clinical relevance of cluster analysis in phenotyping allergic rhinitis in a real-life study. Int Arch Allergy Immunol. 2015;166(3):231240. DOI: http://dx.doi.org/10.1159/000381339

20. Del-Cuvillo A, Montoro J, Bartra J, Valero A, Ferrer M, Jauregui I, et al. Validation of ARIA duration and severity classifications in Spanish allergic rhinitis patients-The ADRIAL cohort study. Rhinology. 2010;48(2):201-205. DOI: http://dx.doi.org/10.4193/Rhin09.099

21. Calderon MA, Demoly P, Casale T, Akdis CA, Bachert C, Bewick M, et al. Allergy immunotherapy across the life cycle to promote active and healthy ageing: from research to policies: An AIRWAYS Integrated Care Pathways (ICPs) programme item (Action Plan B3 of the European Innovation Partnership on Active and Healthy Ageing) and the Global Alliance against Chronic Respiratory Diseases (GARD), a World Health Organization GARD research demonstration project. Clin Transl Allergy. 2016;6:41. DOI: http://dx.doi.org/10.1186/s13601-016-0131-x

22. Cruz AA, Popov T, Pawankar R, Annesi-Maesano I, Fokkens W, Kemp J, et al. Common characteristics of upper and lower airways in rhinitis and asthma: ARIA update, in collaboration with GA(2)LEN. Allergy. 2007;62 Suppl 84:1-41. DOI: http://dx.doi.org/10.1111/j.1398-9995.2007.01551.x

23. Bousquet PJ, Devillier P, Tadmouri A, Mesbah K, Demoly P, Bousquet J. Clinical relevance of cluster analysis in phenotyping allergic rhinitis in a real-life study. Int Arch Allergy Immunol. 2015;166(3):231240. DOI: http://dx.doi.org/10.1159/000381339

24. Meltzer EO, Wallace D, Dykewicz M, Shneyer L. Minimal Clinically Important Difference (MCID) in allergic rhinitis: Agency for healthcare research and quality or anchor-based thresholds. J Allergy Clin Immunol Pract. 2016;4(4):682-688. DOI: http://dx.doi.org/10.1016/j.jaip.2016.02.006

25. Bousquet J, Schunemann HJ, Fonseca J, Samolinski B, Bachert C, Canonica GW, et al. MACVIA-ARIA Sentinel Network for allergic rhinitis (MASK-rhinitis): The new generation guideline implementation. Allergy. 2015;70(11):1372-1392. DOI: http://dx.doi.org/10.1111/all.12686

26. Bourret R, Bousquet J, Mercier J, Camuzat T, Bedbrook A, Demoly P, et al. MASK-rhinitis, a single tool for integrated care pathways in allergic rhinitis. World Hosp Health Serv. 2015;51(3):36-39.

27. Bousquet J, Schünemann HJ, Hellings PW, Arnavielhe S, Bachert C, Bedbrook A, et al. MACVIA clinical decision algorithm in adolescents and adults with allergic rhinitis. J Allergy Clin Immunol. 2016;138(2):367-374. DOI: http://dx.doi.org/10.1016/j.jaci.2016.03.025

28. Bousquet J, Caimmi D, Bedbrook A, Bewick M, Hellings P, Devillier P, et al. Pilot study of mobile phone technology in allergic rhinitis in European countries: the MASK-rhinitis study. Allergy. 2017;72(6):857865. DOI: http://dx.doi.org/10.1111/all.13125

29. König HH, Bernert S, Angermeyer MC, Matschinger H, Martinez M, Vilagut G, et al. Comparison of population health status in six European countries: Results of a representative survey using the EQ-5D questionnaire. Med Care. 2009;47(2):255-261. DOI: http://dx.doi.org/10.1097/MLR.0b013e318184759e

30. Blanc PD, Trupin L, Eisner M, Earnest G, Katz PP, Israel L, et al. The work impact of asthma and rhinitis: findings from a population-based survey. J Clin Epidemiol. 2001;54(6):610-618. DOI: http://dx.doi. org/10.1016/S0895-4356(00)00349-8

31. Bousquet J, Bachert C, Canonica GW, Casale TB, Cruz AA, Lockey RJ, et al. Unmet needs in severe chronic upper airway disease (SCUAD). J Allergy Clin Immunol. 2009;124(3):428-433. DOI: http://dx.doi. org/10.1016/j.jaci.2009.06.027

32. Bousquet J, Anto J, Sunyer J, Nieuwenhuijsen M, Vrijheid M, Keil T, et al. Pooling birth cohorts in allergy and asthma: European Union-funded initiatives-a MeDALL, CHICOS, ENRIECO, and GA(2)LEN joint paper. Int Arch Allergy Immunol. 2013;161(1):1-10. DOI: http://dx.doi.org/10.1159/000343018

33. Westman M, Lupinek C, Bousquet J, Andersson N, Pahr S, Baar A, et al. Early childhood IgE reactivity to pathogenesis-related class 10 proteins predicts allergic rhinitis in adolescence. J Allergy Clin Immunol. 2015;135(5):1199-1206. DOI: http://dx.doi.org/10.1016/j.jaci.2014.10.042

34. Bousquet J, Anto JM, Akdis M, Auffray C, Keil T, Momas I, et al. Paving the way of systems biology and precision medicine in allergic diseases: The MeDALL success story: Mechanisms of the Development of 
Ivancevich JC et al. ARIA 2016: vías integradas de atención para la medicina predictiva

ALLergy; EU FP7-CP-IP; Project No: 261357; 2010-2015. Allergy. 2016;71(11):1513-1525. DOI: http:// dx.doi.org/10.1111/all.12880

35. Burte E, Bousquet J, Varraso R, Gormand F, Just J, Matran R, et al. Characterization of rhinitis according to the asthma status in adults using an unsupervised approach in the EGEA Study. PLoS One. 2015;10(8):e0136191. DOI: http://dx.doi.org/10.1371/journal.pone.0136191

36. Siroux V, Lupinek C, Resch Y, Curin M, Just J, Keil T, et al. Specific IgE and IgG measured by the MeDALL allergen-chip depend on allergen and route of exposure: The EGEA study. J Allergy Clin Immunol. 2017;139(2):643-654. DOI: http://dx.doi.org/10.1016/j.jaci.2016.05.023

37. Just J, Elegbede CF, Deschildre A, Bousquet J, Moneret-Vautrin DA, Crepet A, et al. Three peanutallergic/sensitized phenotypes with gender difference. Clin Exp Allergy. 2016;46(12):1596-1604. DOI: http://dx.doi.org/10.1111/cea.12791

38. Zoratti EM, Krouse RZ, Babineau DC, Pongracic JA, O'Connor GT, Wood RA, et al. Asthma phenotypes in inner-city children. J Allergy Clin Immunol. 2016;138(4):1016-1029. DOI: http://dx.doi.org/10.1016/j. jaci.2016.06.061

39. Bresciani M, Paradis L, Des Roches A, Vernhet H, Vachier I, Godard P, et al. Rhinosinusitis in severe asthma. J Allergy Clin Immunol. 2001;107(1):73-80. DOI: http://dx.doi.org/10.1067/mai.2001.111593

40. Ten-Brinke A, Grootendorst DC, Schmidt JT, De-Bruïne FT, Van-Buchem MA, Sterk PJ, et al. Chronic sinusitis in severe asthma is related to sputum eosinophilia. J Allergy Clin Immunol. 2002;109(4):621626. DOI: http://dx.doi.org/10.1067/mai.2002.122458 